\title{
A characteristic biosignature for discrimination of gastric cancer from healthy population by high throughput GC-MS analysis
}

\author{
Yinan Chen ${ }^{1, *}$, Jun Zhang ${ }^{1, *}$, Lei Guo ${ }^{1}$, Lei Liu ${ }^{1}$, Jingran Wen ${ }^{2}$, Lu Xu ${ }^{1}$, Min Yan ${ }^{1}$, \\ Zuofeng $\mathrm{Li}^{2}$, Xiaoyan Zhang ${ }^{2}$, Peng $\mathrm{Nan}^{3}$, Jinling Jiang ${ }^{1}$, Jun $\mathrm{Ji}^{1}$, Jianian Zhang ${ }^{1}$, \\ Wei Cai ${ }^{1}$, Huisheng Zhuang ${ }^{4}$, Yan Wang ${ }^{5}$, Zhenggang Zhu ${ }^{1}$ and Yingyan Yu ${ }^{1}$ \\ ${ }^{1}$ Department of Surgery of Ruijin Hospital, and Shanghai Institute of Digestive Surgery, Shanghai Key Laboratory for Gastric \\ Neoplasms, Ruijin Hospital, Shanghai Jiao Tong University School of Medicine, Shanghai, China \\ ${ }^{2}$ Tongji University, School of Life Science and Technology, Shanghai, China \\ ${ }^{3}$ School of Life Sciences, Fudan University, Shanghai, China \\ ${ }^{4}$ School of Environmental Science and Engineering, Shanghai Jiao Tong University, Shanghai, China \\ ${ }^{5}$ College of Public Health, Shanghai Jiao Tong University, School of Medicine, Shanghai, China \\ * These authors have contributed equally to this work \\ Correspondence to: Yingyan Yu, email: yingyan3y@sjtu.edu.cn
}

Zhenggang Zhu, email: zzg1954@hotmail.com

Keywords: gastric cancer; urine; metabolomics; biomarkers; GC-MS

Received: March 29, $2016 \quad$ Accepted: July 19, 2016

Published: August 31, 2016

\section{ABSTRACT}

Early diagnosis of gastric cancer is crucial to improve patient' outcome. A good biomarker will function in early diagnosis for gastric cancer. In order to find practical and cost-effective biomarkers, we used gas chromatography combined mass spectrometer (GC-MS) to profile urinary metabolites on 293 urine samples. Ninetyfour samples are taken as training set, others for validating study. Orthogonal partial least squares discriminant analysis (OPLS-DA), significance analysis of microarray (SAM) and Mann-Whitney $U$ test are used for data analysis. The diagnostic value of urinary metabolites was evaluated by ROC curve. As results, Seventeen metabolites are significantly different between patients and healthy controls in training set. Among them, 14 metabolites show diagnostic value better than classic blood biomarkers by quantitative assay on validation set. Ten of them are amino acids and four are organic metabolites. Importantly, proline, p-cresol and 4-hydroxybenzoic acid disclose outcome-prediction value by means of survival analysis. Therefore, the examination of urinary metabolites is a promising noninvasive strategy for gastric cancer screening.

\section{INTRODUCTION}

Gastric cancer ranks the fifth in incidence and the third in mortality among all cancers worldwide [1]. Since there are no specific symptoms at early stage of carcinogenesis, most of patients are diagnosed at advanced stage with lymph node or remote metastasis. Therefore, exploring valuable molecular characterization or biomarkers is desirable for improving current status.

Metabolomics focuses on exploring low-molecular weight metabolites in a biological system [2]. Metabolites are the end products of life activity and always present in body fluids, which are relatively easier to get samples. However, the amount of metabolites is less than that of genes and proteins in cells. For example, Saccharomyces cerevisiae contains over 6000 genes but only 600 metabolites [3]. So it is relatively easy to find out biosignatures from metabolites profile analysis. Metabolites analysis has been applied to the diagnosis of many cancers such as gastric cancer $[4,5]$, breast cancer [6-9], ovarian cancer [10], pancreatic cancer [11], colorectal cancer [12-15], prostate cancer [16, 17], liver cancer [18, 19], lung cancer [20-23] and bladder cancer $[24,25]$. There have been several metabolomics studies on gastric cancer with various platforms such as CE-MS [26], LC-MS [27] and NMR [4, 28]. Up-to-date, most of 
the studies analyzed small sample sizes and are lack of further validation on a separated cohort. Moreover, none of them could predict the prognosis of gastric cancer. In order to satisfy the clinical demand, more metabolites analysis on large sample size is expected.

GC-MS is widely applied in metabolomics study, which provide a rapid, qualitative and quantitative analyses with the ability to identify small molecule metabolites $[29,30]$. GC can separate volatile and thermal stable compounds, then the compounds are eluted and detected by MS. MS is operated by ion formation, separation and detection of ions according to mass-to-charge $(\mathrm{m} / \mathrm{z})$ ratio. Qiu and coworkers [29] applied GC-MS analysis on urine detection with ethyl chloroformate (ECF) derivatization method, which was proved stable and repeatable. In the present study, we described a GC-MS-based urinary metabolites profile on a large cohort of gastric cancer.
By a precise quantitative assay, we characterized a group of metabolites, which disclosed a diagnostic value for gastric cancer, which are better than that of classic blood tumor biomarkers. Three of metabolites could predict patient' outcome. The urinary characteristic metabolites may function as promising noninvasive biomarkers/ biosignature for gastric cancer screening.

\section{RESULTS}

\section{Identification of characteristic urinary metabolites on training set}

A total of 129 metabolites were identified in training set (Supplementary table 1). The peaks showed high

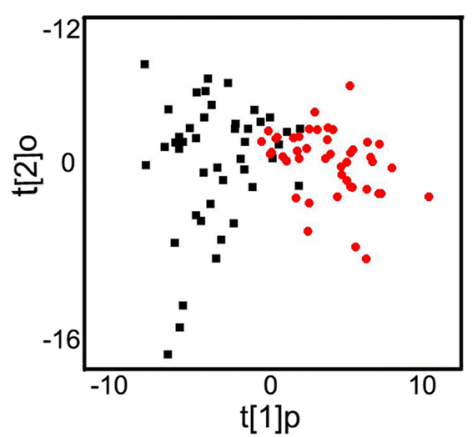

A

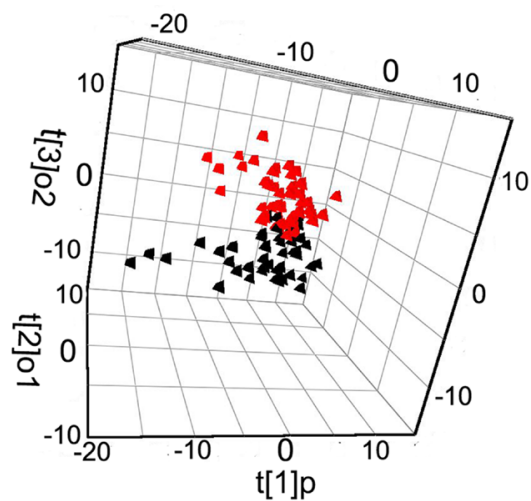

B

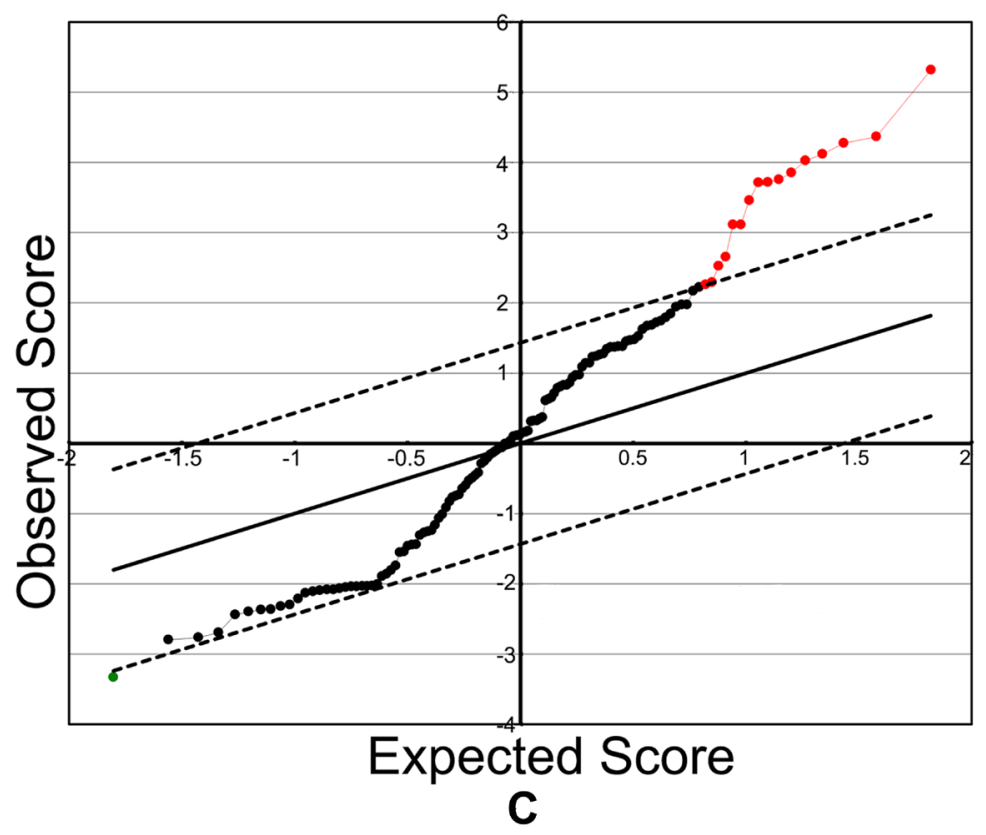

Figure 1: OPLS-DA score plots and SAM analysis plots. A. OPLS-DA score plots. B. OPLS-DA 3D scatter plots. Each symbol represents the metabolomic profile of an individual. The black points represent the controls while the red boxes represent gastric cancer sample. C. The 17 variables selected by SAM analysis. The up-regulated variables in cancer group were presented as red spots on the right top, and one down-regulated variable in cancer group was presented as green spot on the left bottom. 
Table 1: The characteristic urinary metabolites selected by OPLS-DA model, SAM analysis and Mann-Whitney $\mathrm{U}$ test for discriminating gastric cancer from healthy controls

\begin{tabular}{|l|l|l|l|}
\hline Metabolites (OPLS-DA) & VIP & SAM* & Mann-Whitney U* \\
\hline Up-regulated & & & \\
\hline Serine & 1.91 & $\bullet$ & $*$ \\
\hline Isoleucine & 1.90 & $\bullet$ & $*$ \\
\hline Proline & 1.88 & $\bullet$ & $*$ \\
\hline Propanedioic acid & 1.85 & & $*$ \\
\hline Phenylalanine, carbamic acid, & 1.80 & $\bullet$ & $*$ \\
\hline Tryptophan & 1.72 & $\bullet$ & $*$ \\
\hline Ethyl 2-methylacetoacetate & 1.72 & $\bullet$ & $*$ \\
\hline 2,3-Octanedione & 1.70 & $\bullet$ & $*$ \\
\hline Glycine & 1.69 & $\bullet$ & $*$ \\
\hline Levulinic acid & 1.63 & $\bullet$ & $*$ \\
\hline 4-Hydroxybenzoic acid & 1.61 & $\bullet$ & $*$ \\
\hline Valine & 1.60 & $\bullet$ & $*$ \\
\hline Tryptamine & 1.55 & & $*$ \\
\hline Benzil & 1.54 & $\bullet$ & $*$ \\
\hline Threonine & 1.54 & $\bullet$ & $*$ \\
\hline Tyrosine & 1.53 & $\bullet$ & $*$ \\
\hline p-cresol & 1.53 & & $*$ \\
\hline Butanedioic acid, ethylidene- & 1.49 & & $*$ \\
\hline Alanine & 1.46 & $\bullet$ & $*$ \\
\hline Methionine & 1.39 & $\bullet$ & $*$ \\
\hline Benzylmalonic acid & 1.32 & & $*$ \\
\hline Down-regulated & & & \\
\hline 5-methyl-hexene & 1.43 & & $*$ \\
\hline 2,2,6,6-tetramethyl-4-oximinopiperdin-1-oxyl & 1.39 & & $*$ \\
\hline Carbamic acid, ethyl & 1.40 & & $*$ \\
\hline Hippuric acid & 1.33 & $\bullet$ & $*$ \\
\hline 3-Pyridinecarboxamide & 1.23 & & $*$ \\
\hline (n) & $\bullet$ & \\
\hline
\end{tabular}

*The black point means that the corresponding metabolite was chosen by SAM-analysis. The black star means that the corresponding metabolite differs significantly between healthy control and gastric cancer patient by Mann-Whitney U test.

qualities of the raw data with evenly dispersed retention time. By Orthogonal partial least squares discriminant analysis (OPLS-DA), we obtained one predictive component and two orthogonal components (R2Ycum $=0.631 ; \mathrm{Q} 2$ Ycum $=0.509)$, which could separate the cancer patients from the healthy controls (Figure1A, 1B). The differential metabolites were identified with the criteria of variable importance in the project values (VIP) $>1$. In addition, SAM method and Mann-Whitney $\mathrm{U}$ test were also used for metabolites selection. For instance, 17 metabolites were characterized between two groups by SAM method (Figure 1C), which were well overlapped with those identified in OPLS-DA model. At SAM method, 16 metabolites are increased metabolites, and one is decreased metabolite in cancer group (Table
1). By Mann-Whitney $U$ test, the candidate metabolites showed statistic significance $(P<0.01)$. The metabolites phenylalanine, carbamic acid and 2, 3-octanedione were excluded because of low match percentage with standard mass spectrum. Metabolites p-cresol and benzylmalonic acid were enrolled as their VIP $>1$ in OPLS-DA model and were assumed to be associated with environmental pollution. A total of 17 metabolites were determined as candidate molecules for further validation. They were alanine, glycine, isoleucine, valine, proline, serine, threonine, methionine, tyrosine, tryptophan, hippuric acid, ethyl 2-methylacetoacetate, levulinic acid, benzylmalonic acid, 4-hydroxybenzoic acid, p-cresol and benzil. 
Table 2: The chemicals, retention times, linear range of concentration and linear fitting coefficients of 17 standard chemicals

\begin{tabular}{|c|c|c|c|}
\hline Chemicals & Retention time(min) & Linear range $(\mu \mathrm{g} / 600 \mu \mathrm{l})$ & r2* \\
\hline \multicolumn{4}{|l|}{ Amino acids } \\
\hline L-Alanine & 8.582 & $0.02-40$ & 0.999522 \\
\hline Glycine & 8.668 & $0.02-40$ & 0.999713 \\
\hline L-Valine & 10.567 & $0.02-40$ & 0.999729 \\
\hline L-Serine & 12.197 & $0.50-40$ & 0.997635 \\
\hline L-Isoleucine & 12.228 & $0.02-40$ & 0.999611 \\
\hline L-Threonine & 12.343 & $0.02-20$ & 0.998707 \\
\hline L-Proline & 12.742 & $0.02-40$ & 0.999963 \\
\hline L-Methionine & 17.536 & $0.002-4$ & 0.999849 \\
\hline L-Tyrosine & 31.984 & $0.02-40$ & 0.999713 \\
\hline L-Tryptophan & 33.986 & $0.02-40$ & 0.999409 \\
\hline \multicolumn{4}{|l|}{ Organic acids } \\
\hline Ethyl 2-methylacetoacetate & 4.602 & $0.002-2$ & 0.99963 \\
\hline Levulinic acid & 5.515 & $0.002-4$ & 0.998062 \\
\hline p-cresol & 9.652 & $0.002-4$ & 0.999038 \\
\hline Benzylmalonic acid & 16.391 & $0.002-4$ & 0.999243 \\
\hline 4-Hydroxybenzoic acid & 17.348 & $0.002-4$ & 0.999731 \\
\hline Hippuric acid & 18.586 & $0.1-40$ & 0.998476 \\
\hline Benzil & 19.580 & $0.002-2$ & 0.996025 \\
\hline
\end{tabular}

*Regression coefficient

Table 3: Recovery of the 17 standards

\begin{tabular}{|l|l|l|}
\hline Chemicals & Recovery (\%)* & \\
\hline & Average & R.S.D \\
\hline Alanine & 92.6 & 4.0 \\
\hline Glycine & 86.9 & 3.6 \\
\hline Valine & 85.6 & 5.2 \\
\hline Isoleucine & 98.6 & 7.0 \\
\hline Serine & 94.5 & 3.8 \\
\hline Threonine & 96.6 & 4.9 \\
\hline Proline & 88.8 & 4.2 \\
\hline Methionine & 83.8 & 5.3 \\
\hline Tyrosine & 81.1 & 5.0 \\
\hline Tryptophan & 85.5 & 4.5 \\
\hline Ethyl 2-methylacetoacetate & 87.3 & 3.53 \\
\hline Levulinic acid & 89.0 & 4.0 \\
\hline p-cresol & 85.9 & 4.4 \\
\hline Benzylmalonic acid & 98.5 & 8.7 \\
\hline 4-Hydroxybenzoic acid & 87.7 & 3.6 \\
\hline Hippuric acid & 104.7 & 7.91 \\
\hline Benzil & 107.3 & 3.8 \\
\hline
\end{tabular}

*Mean recovery was obtained by 16 determinations (four parallel samples at four different concentrations) with an internal standard.

\section{Validation of 17 selected metabolites in validating set}

We quantitatively examined the levels of 17 metabolites in urine samples, including 10 amino acids and 7 organic small molecules. A total of 199 urine samples were enrolled in the study. Of them, 112 samples were from gastric cancer (including 37 cases of early gastric cancer), and 87 were healthy controls. The candidate metabolites were validated through external standard method. The 
chemicals, retention time, linear range of concentration and linear fitting coefficients of 17 standard chemicals are summarized in Table 2. The total ion current (TIC) chromatogram of the standard chemicals was presented in Figure 2A.The TIC chromatograms of urine samples derived from the healthy controls and gastric cancer are obtained by GC-MS analysis. The peaks of 17 compounds were identified by the spectrums of the known standards (Supplementary Figure 1, 2, 3). The amount of metabolites depended on the efficiency of chemical derivatization. We randomly detected twice for one sample by two different operators at interval of 48 hours and found that the two TIC profiles of same sample were overlapped well, which reflected the stability and reliability of GC-MS analysis (Figure 2B, 2C and 2D). We also tested the recovery of standards in urine to validate our methodology to analyze complex compounds. A $300 \mu \mathrm{l}$ standard solution with 5, 10,20 and $40 \mu \mathrm{g} / \mathrm{ml}$ of 17 compounds was spiked to $300 \mu \mathrm{l}$ urine, prior to ECF-derivatization. The extraction recovery was calculated and the mean recovery of 17 compounds with different concentrations ranged from 81.1 to $107.3 \%$ with the relative standard derivation (R.S.D) lower than 9\% (Table 3). By TIC analysis, gastric cancer disclosed significant difference from healthy control (Figure 3A, 3B). This difference was more distinct at enlarged TIC chromatogram from 7.9 to $13.1 \mathrm{~min}$ (Figure 3C).

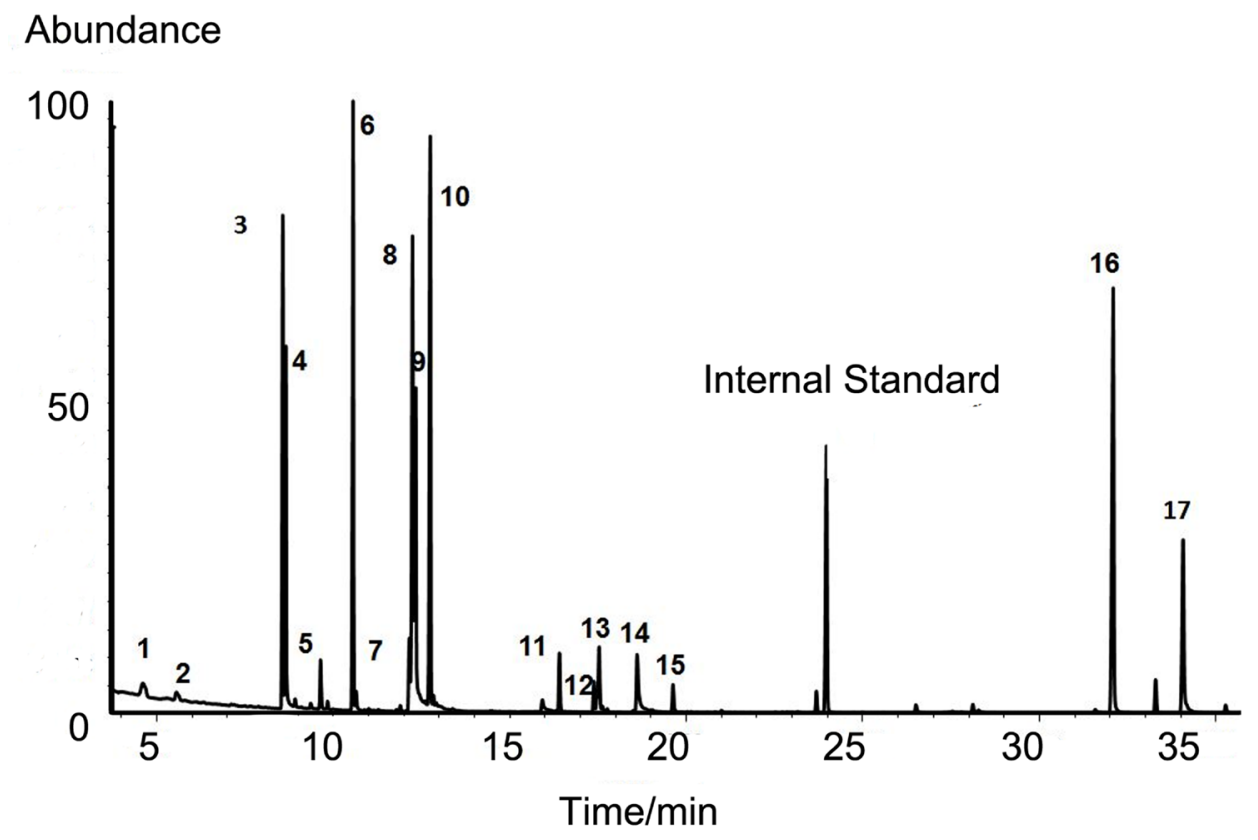

A
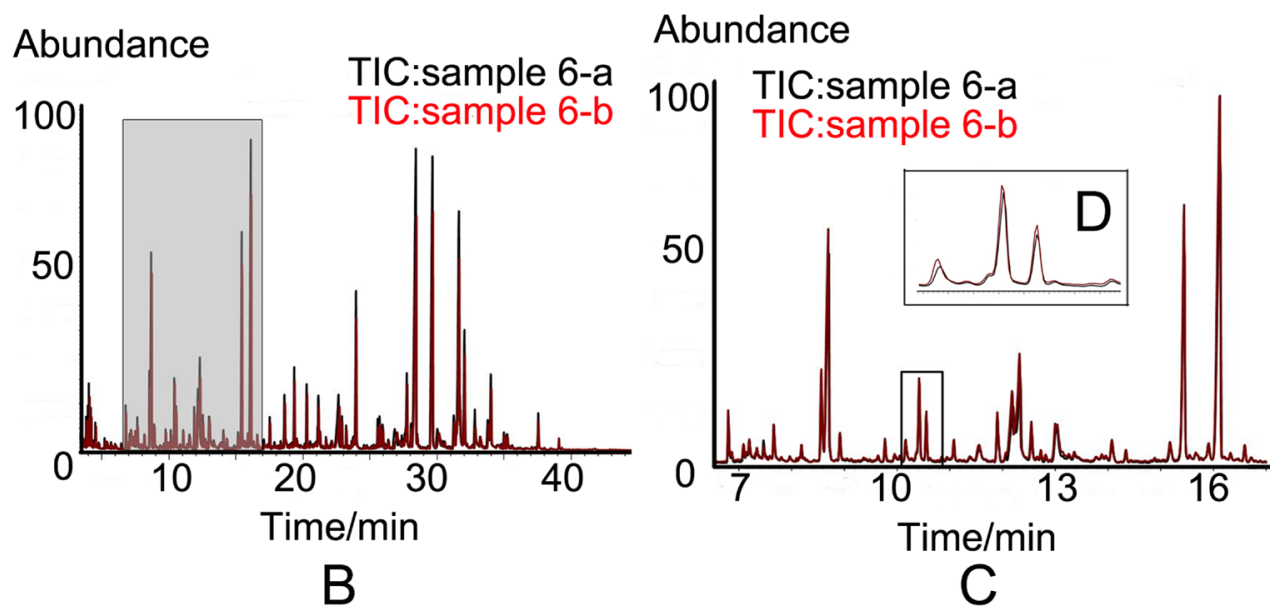

Figure 2: TIC chromatogram. A. TIC chromatogram of the 17 candidate standards by GC-MS. The peak numbers from 1 to 17 represent ethyl 2-methylacetoacetate, levulinic acid, alanine, glycine, p-cresol, valine, serine, isoleucine, threonine, proline, benzylmalonic acid, 4-hydroxybenzoic acid, methionine, hippuric acid, benzil, tyrosine and tryptophan. L-2-chlorophenylalanine was used as an internal quality standard. B. The overlay of two TIC chromatograms from one sample. C. Enlargement of TIC from 7 to 17 -min. D. Further enlargement of regional peaks. 
Table 4:The median and interquartile range $(\mathrm{IQR})$ of urinary metabolite levels $(\mu \mathrm{g} / \mathrm{ml})$

\begin{tabular}{|l|l|l|l|l|l|}
\hline Metabolite & Healthy & Cancer & $\boldsymbol{P}$ value & Early cancer & $\boldsymbol{P}$ value \\
\hline Alanine & $12.615(11.553)$ & $24.514(16.555)$ & $<0.001$ & $23.794(12.689)$ & $<0.001$ \\
\hline Glycine & $42.042(42.262)$ & $63.654(50.772)$ & $<0.001$ & $63.490(46.492)$ & $<0.001$ \\
\hline Valine & $2.770(2.486)$ & $4.090(2.333)$ & $<0.001$ & $4.150(1.760)$ & $<0.001$ \\
\hline Isoleucine & $1.243(1.047)$ & $1.813(1.207)$ & $<0.001$ & $1.783(0.717)$ & $<0.001$ \\
\hline Serine & $22.351(18.974)$ & $39.046(24.530)$ & $<0.001$ & $38.926(26.711)$ & $<0.001$ \\
\hline Threonine & $6.573(4.820)$ & $11.162(7.503)$ & $<0.001$ & $11.556(5.973)$ & $<0.001$ \\
\hline Proline & $0.450(0.373)$ & $0.810(0.540)$ & $<0.001$ & $0.717(0.510)$ & $<0.001$ \\
\hline Methionine & $0.797(0.843)$ & $1.500(0.983)$ & $<0.001$ & $1.567(0.837)$ & $<0.001$ \\
\hline Tyrosine & $7.969(9.202)$ & $12.062(7.153)$ & $<0.001$ & $11.689(5.289)$ & 0.002 \\
\hline Tryptophan & $9.599(8.243)$ & $13.489(8.949)$ & $<0.001$ & $13.209(7.586)$ & 0.004 \\
\hline Molecule 1 & $0.803(0.683)$ & $1.200(0.917)$ & $<0.001$ & $1.167(0.727)$ & $<0.001$ \\
\hline Molecule 2 & $0.450(0.577)$ & $0.747(0.660)$ & $<0.001$ & $0.793(0.590)$ & 0.001 \\
\hline Molecule 3 & $0.040(0.033)$ & $0.070(0.093)$ & $<0.001$ & $0.053(0.060)$ & 0.047 \\
\hline Molecule 4 & $0.107(0.113)$ & $0.137(0.160)$ & $<0.001$ & $0.143(0.123)$ & 0.013 \\
\hline
\end{tabular}

Molecules 1 to 4 represent ethyl 2-methylacetoacetate, levulinic acid, p-cresol and benzylmalonic acid, respectively.

Table 5: The sensitivity, specificity, Youden index, cutoff value, and AUC of each metabolite

\begin{tabular}{|l|l|l|l|l|l|}
\hline Metabolite & Sensitivity & Specificity & Youden & Cutoff & AUC \\
\hline Alanine & 0.783 & 0.678 & 0.461 & 18.268 & 0.804 \\
\hline Glycine & 0.915 & 0.414 & 0.329 & 35.603 & 0.744 \\
\hline Valine & 0.623 & 0.735 & 0.358 & 3.586 & 0.734 \\
\hline Isoleucine & 0.670 & 0.724 & 0.394 & 1.517 & 0.770 \\
\hline Serine & 0.726 & 0.759 & 0.485 & 30.227 & 0.814 \\
\hline Threonine & 0.811 & 0.678 & 0.489 & 8.016 & 0.823 \\
\hline Proline & 0.840 & 0.632 & 0.472 & 0.560 & 0.793 \\
\hline Methionine & 0.679 & 0.782 & 0.461 & 1.260 & 0.784 \\
\hline Tyrosine & 0.858 & 0.472 & 0.330 & 7.179 & 0.693 \\
\hline Tryptophan & 0.821 & 0.517 & 0.338 & 9.676 & 0.698 \\
\hline Molecule 1 & 0.538 & 0.804 & 0.342 & 1.163 & 0.715 \\
\hline Molecule 2 & 0.651 & 0.644 & 0.295 & 0.583 & 0.673 \\
\hline Molecule 3 & 0.726 & 0.621 & 0.347 & 0.043 & 0.698 \\
\hline Molecule 4 & 0.472 & 0.839 & 0.311 & 0.177 & 0.669 \\
\hline Combination & 0.774 & 0.851 & 0.625 & 0.555 & 0.893 \\
\hline
\end{tabular}

Molecules 1 to 4 represent ethyl 2-methylacetoacetate, levulinic acid, p-cresol, benzylmalonic acid, respectively.

Table 6: Baseline characteristics of the samples.

\begin{tabular}{|l|l|l|l|l|}
\hline \multirow{2}{*}{ Characteristic } & Training set & \multicolumn{2}{l|}{ Validating set } \\
\cline { 2 - 5 } & Healthy & Cancer & Healthy & Cancer \\
\hline Number & 47 & 47 & 87 & 112 \\
\hline Age(median, range) & $57 / 25-80$ & $55 / 22-78$ & $58 / 24-83$ & $59 / 27-87$ \\
\hline Male/female ratio & $25 / 22$ & $27 / 20$ & $46 / 41$ & $61 / 51$ \\
\hline TNM stage & - & 13 & - & 37 \\
\hline StageIa/b & - & 12 & - & 23 \\
\hline StageIIa/b & - & 10 & - & 24 \\
\hline StageIIIa/b/c & - & 12 & - & 28 \\
\hline StageIV & - & & \multicolumn{5}{l}{} \\
\hline
\end{tabular}




\section{Diagnostic value analysis of 17 urinary metabolites}

By Mann-Whitney U test, gastric cancer group could be clearly discriminated by candidate metabolites except for 4-hydroxybenzoic acid, benzil and hippuric acid. This difference was also observed between healthy control and early gastric cancer group. Intensive analysis indicated that the concentration of metabolite p-cresol closely correlates with cancer stage. The levels of p-cresol are gradually increased with patients' stages (Supplementary Figure 4). The median and interquartile range (IQR) of each candidate metabolite in healthy control, gastric cancer group and early gastric cancer group were listed in Table 4. Fourteen out of 17 candidates $(82.35 \%)$ revealed diagnostic value on ROC analysis. Six of them revealed satisfactory diagnostic values with area under ROC curve (AUC) more than 0.75 . The box charts of these 6 urinary

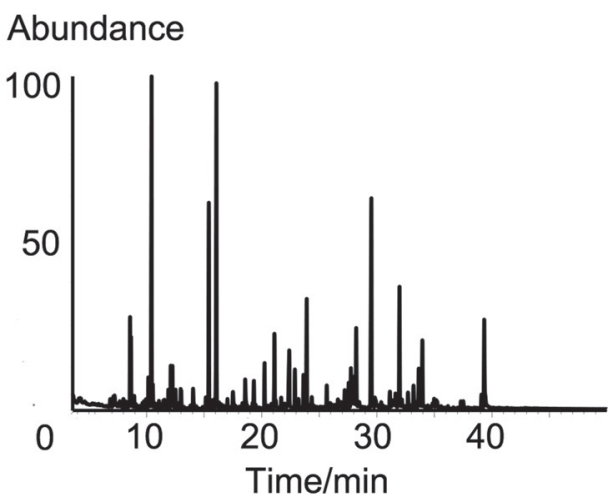

B

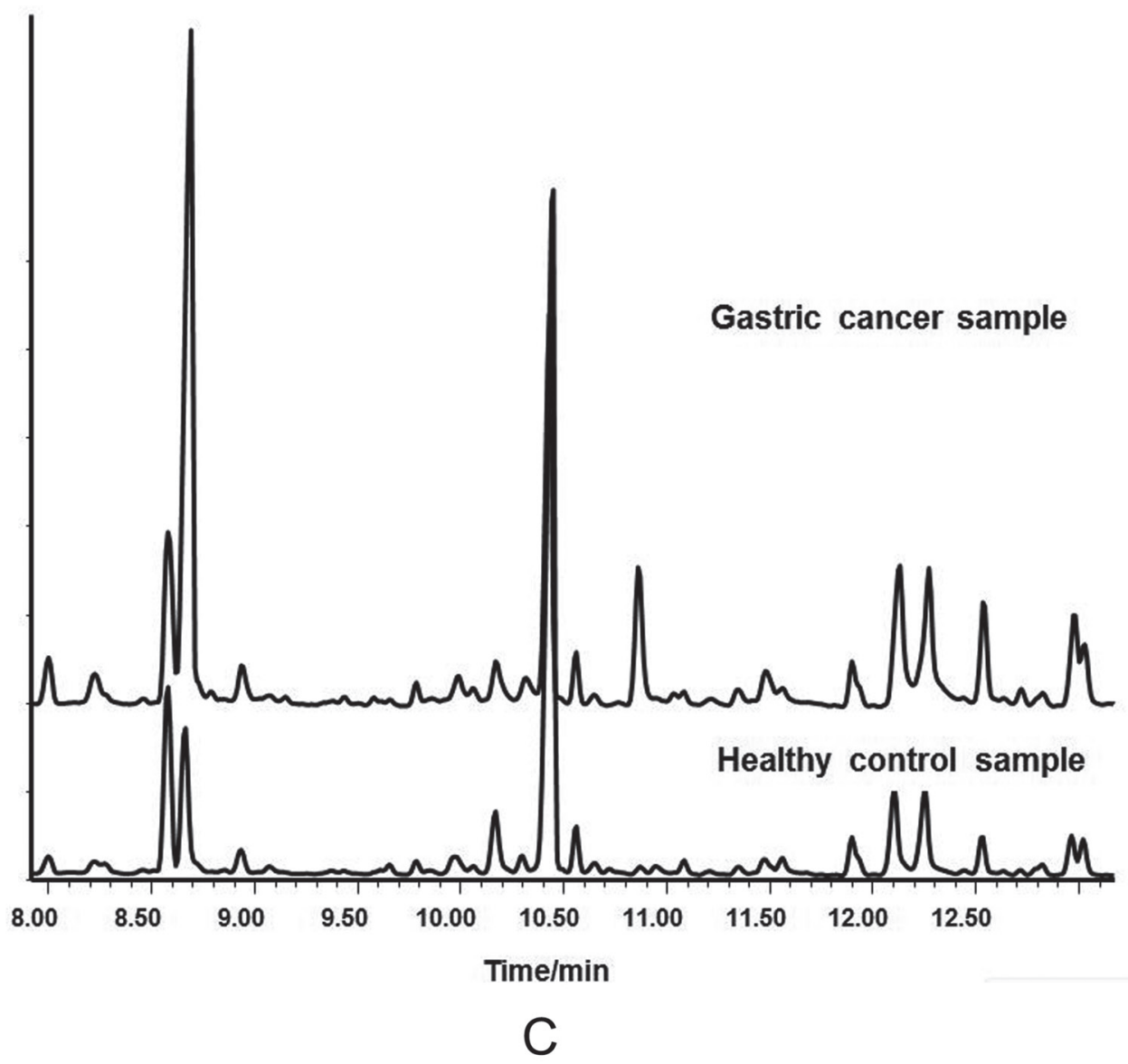

Figure 3: TIC chromatograms of urine samples from different groups by GC-MS analysis. A. TIC chromatogram of healthy control. B. TIC chromatogram of gastric cancer patient. C. Enlargement of TIC chromatogram at 7.9-min to 13.1-min for comparison of healthy control with gastric cancer patient. 
candidate metabolites between healthy controls and gastric cancer groups or early gastric cancer samples are plotted (Figure 4, Figure 5). Their ROC curves were shown in Figure 6A.

The AUC, sensitivity, specificity and Youden value were listed in Table 5. The cutoff value for each metabolite was determined on the basis of the Youden index $[\mathrm{J}, \mathrm{J}$ $=\max \{$ sensitivity + specificity-1\}] [31]. We created logistic regression model by binary logistic regression analysis. Zero (health control) or one (gastric cancer) serves as dichotomous variable, and fourteen differential metabolites as the covariates. The predicted equation was as follows:
$P=1 /[1+\mathrm{e}-(0.222 \mathrm{ala}-0.019 \mathrm{gly}-1.334 \mathrm{val}+2.375$ iso $+0.173 \mathrm{ser}+0.709 \mathrm{thr}+2.844 \mathrm{p}$ ro+4.040met-0.793tyr-0.044try+0.693a+0.417b+14.801c-0.157d-3.853) $]$. The letters a, b, c and d represent ethyl 2-methylacetoacetate, levulinic acid, p-cresol and benzylmalonic acid, respectively. The $P$ referred to the value of predicted probability of each sample based on the levels of 14 candidate metabolites. We used the values of predicted probability as new variables and produced a combined ROC curve with a cutoff value 0.555 . The corresponding sensitivity was 0.774 and specificity was 0.851 . The AUC was 0.893 (Figure 6A).

We further compared diagnostic values of urinary molecules with classic blood tumor biomarkers CEA, CA19-9, CA72-4, CA12-5 and AFP on validating set. The

\section{Health VS Cancer}
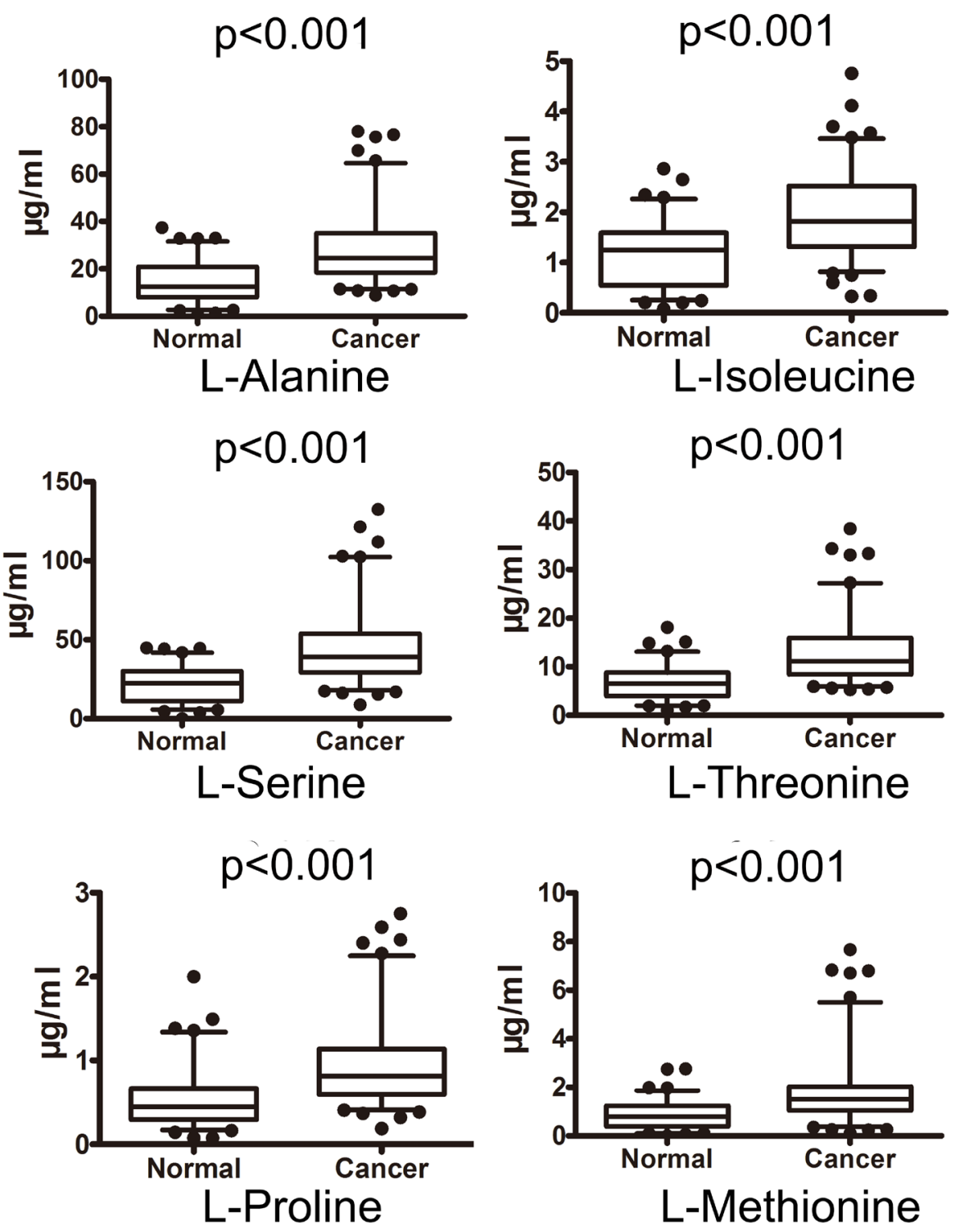

Figure 4: Box plots of the levels of potential urinary biomarkers that could distinguish cancer from controls. The p-values of Mann-Whitney U test were indicated (the concentration unit $\mu \mathrm{g} / \mathrm{ml}$ ). 
raising of any blood tumor biomarkers in one sample was considered as positivity. We established a combined ROC curve of blood tumor biomarkers. We created ROC curves of CEA, CA19-9, CA72-4, CA12-5 and AFP, respectively. The AUC of single blood biomarker ranged from 0.525 to 0.590 . The combination AUC was 0.685 . However, the combined AUC of urinary molecules was 0.810 , which is superior to the combination AUC of classic blood biomarkers (Figure 6B).

\section{Health VS Early}
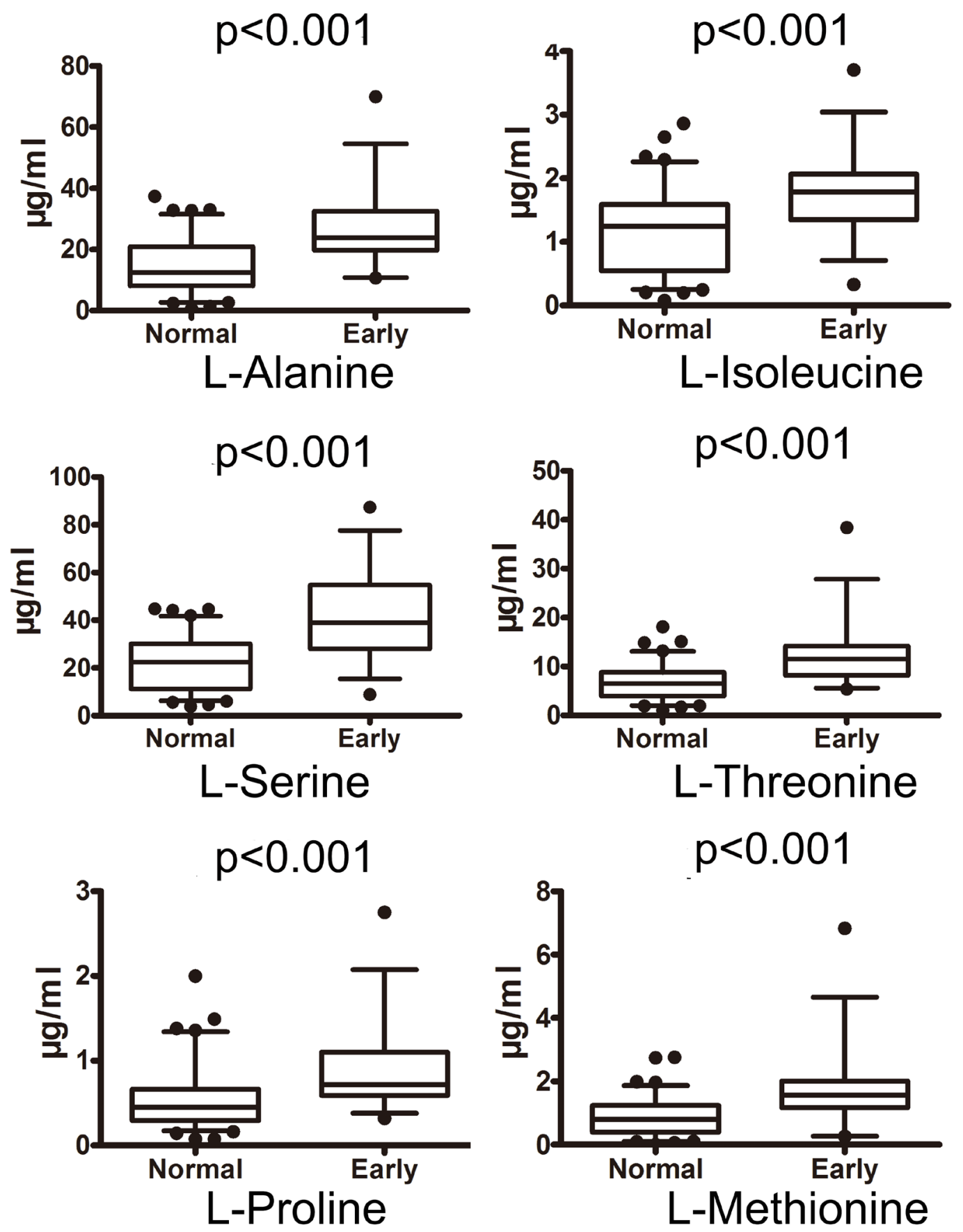

Figure 5: Box plots of the levels of potential urinary biomarkers that could distinguish early gastric cancer from controls. The p-values of Mann-Whitney $U$ test were indicated (the concentration unit $\mu \mathrm{g} / \mathrm{ml}$ ). 
The prognostic value analysis of 17 urinary metabolites

We got follow-up information on 82 out of 112 gastric cancer cases in validating set. The follow-up period was 3 to 5 years after surgery. At the end of follow-up, 35 patients (42.7\%) were alive and 47 patients $(57.3 \%)$ died.
We calculated the survival rate based on metabolites levels using Kaplan-Meier method. The differences in overall survival between two groups were compared by log-rank tests. We found that high levels of proline, p-cresol and 4-hydroxybenzoic acid revealed worse survival rate $(P<$ 0.01 ) with median survival time 16 -mons, 15 -mons and 15 -mons respectively (Figure 6C, 6D and 6E). The median
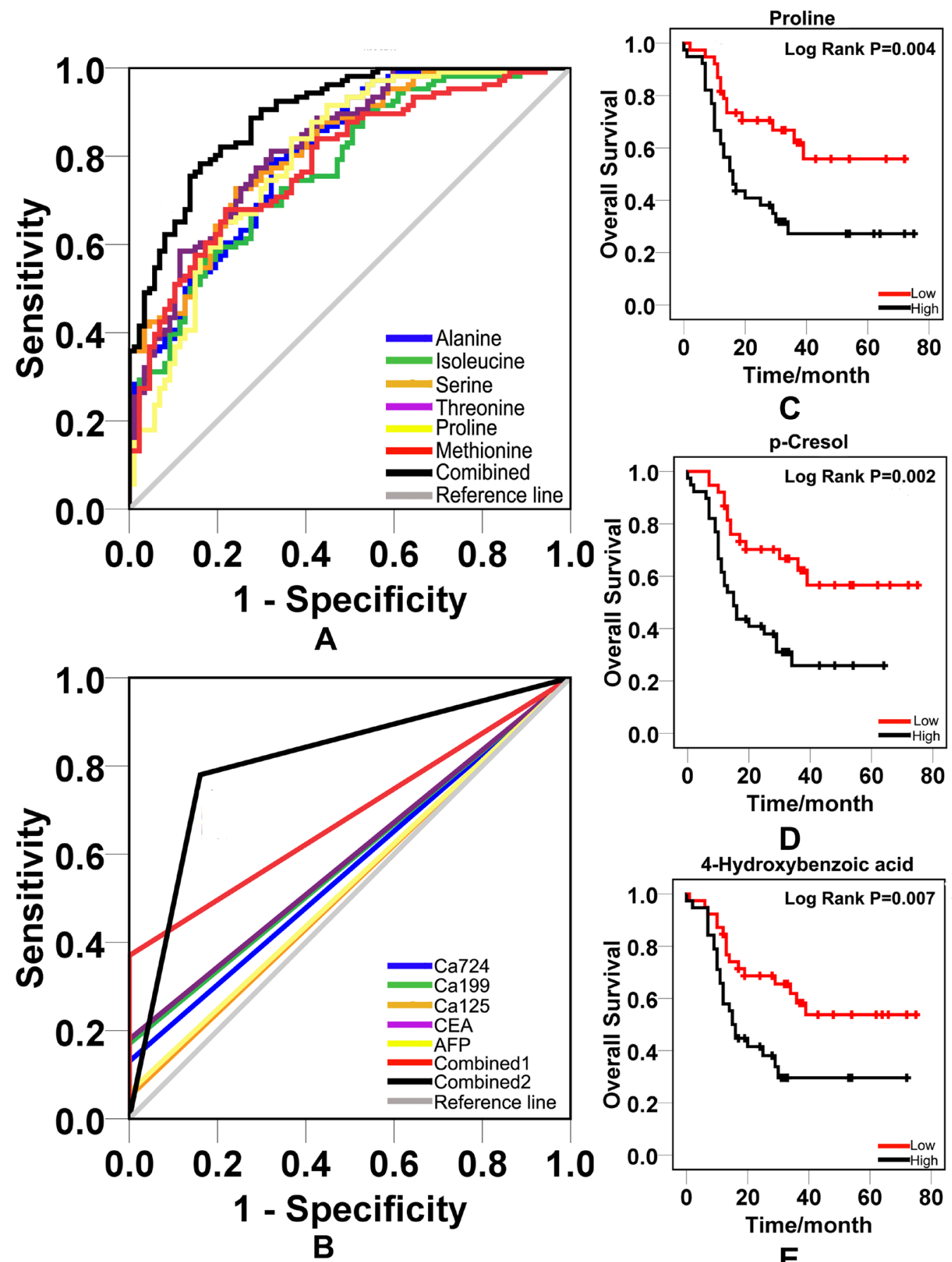

D

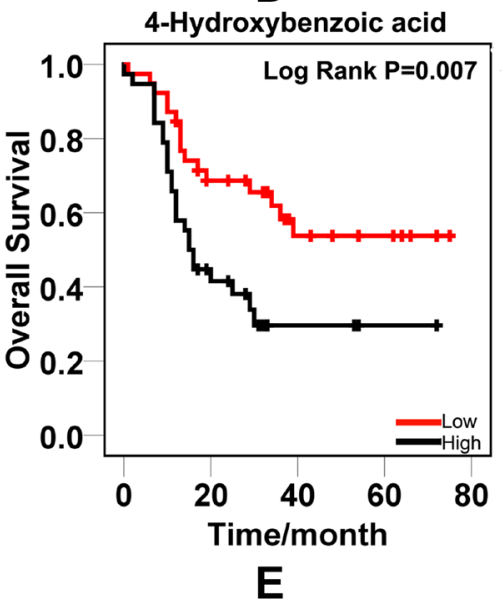

Figure 6: ROC curves and survival curves. A. ROC curves were obtained from 14 increased urinary metabolites and their combination. B. ROC curves were obtained from classic blood biomarkers and their combination. Combined 1 was the combination of classic blood tumor markers. Combined 2 was the combination of 14 new potential urinary biomarkers. C. The survival curve of proline. D. The survival curve of p-cresol. E. The survival curve of 4-hydroxybenzoic acid. 
survival time was over 60 months when patients revealed lower levels of proline, p-cresol and 4-hydroxybenzoic acid in urine. Other metabolites were not associated with the prognosis. By hazard ratio (HR) analysis, the HR of p-cresol, proline and 4-hydroxybenzoic acid was 2.688 (95\% CI: 1.403-5.150), 2.473(95\%CI: 1.293-4.728) and 2.335(95\% CI: 1.229-4.435), respectively.

\section{Metabolic pathway analysis}

We analyzed the relevant metabolic pathways of gastric cancer by MetPA tool. The identified compounds were distributed in 27 pathways (Supplementary Table $2)$. The main metabolic pathways included glycine, serine and threonine metabolism (glycine, serine and threonine), arginine and proline metabolism (proline), cysteine and methionine metabolism (methionine, alanine and serine), valine, leucine and isoleucine biosynthesis (threonine, isoleucine and valine), taurine and hypotaurine metabolism (alanine), alanine, aspartate and glutamate metabolism (alanine) (Figure 7A). We noticed that the pathway of glycine, serine and threonine metabolism were excessively activated in gastric cancer (Figure 7B).

\section{DISCUSSION}

Early diagnosis of gastric cancer is crucial to improve patient' outcome. Once the patient was diagnosed at early stage, he or she will obtain timely treatment [32].

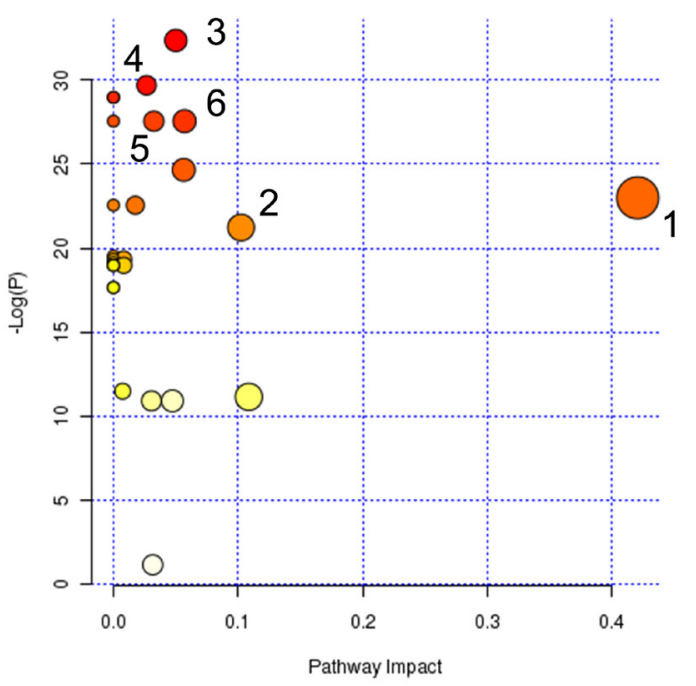

A
Current diagnosis of gastric cancer relies on imaging, endoscopy and histological pathology. Although these examinations are specific and accurate, they are invasive, expensive and therefore not suitable for population screening. Classic blood tumor biomarkers such as CEA, AFP, CA72-4 and CA19-9 are not sensitive enough for gastric cancer diagnosis. Therefore, new diagnostic procedures with better sensitivity and specificity are needed. Here we describe a promising noninvasive procedure. We could obtain the whole metabolites profile on $300 \mu$ urine sample within 40 minutes.

Chan and coworkers ever reported urinary metabolomics of gastric cancer [4]. They identified three discriminatory metabolites, 2-hydroxyisobutyrate, 3-indoxylsulfate and alanine. Another study analyzed 154 urine samples from gastric cancer and healthy controls. They found a group of metabolites related to amino acid and altered lipid metabolism. The sensitivity of metabolites is much higher than that from blood biomarker CA19-9 and CEA [28]. However, the sample sizes of previous studies are not large enough, and the diagnostic capability is limited. Up-to-date, none of the urinary metabolomics studies for gastric cancer involved in patient' prognosis. To our knowledge, this is the largest sample size for urinary metabolomics study on gastric cancer. To characterize the specific metabolites, three rigorous algorithms were used for metabolites selection. We paid attention to metabolites appeared in three algorithms. Finally, 17 metabolites were selected for further validation on validation set. By quantitative

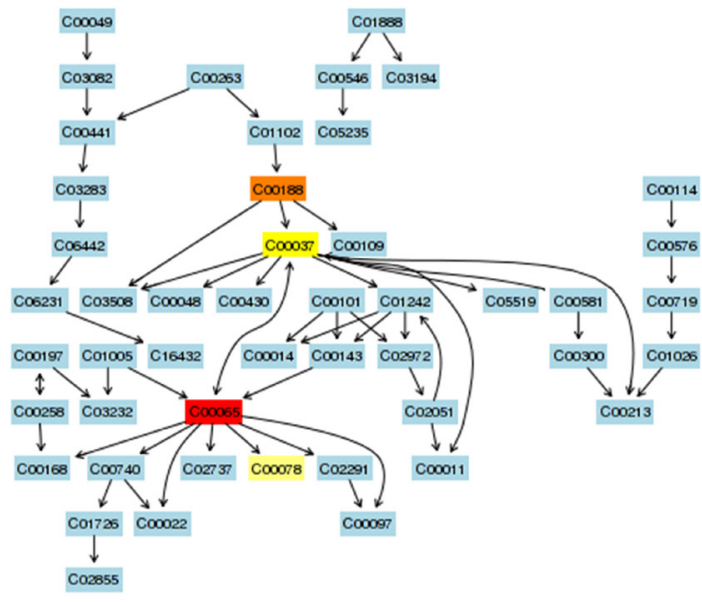

B

Figure 7: The metabolic pathway analysis. A. All enrolled pathways by MetPA analysis. The node color is based on P value and the node radius is determined based on their pathway impact values. 1. glycine, serine and threonine metabolism. 2. arginine and proline metabolism. 3. cysteine and methionine metabolism. 4. Valine, leucine and isoleucine biosynthesis. 5.Taurine and hypotaurine metabolism. 6. Alanine, aspartate and glutamate metabolism. B. The glycine, serine and threonine metabolism pathway. The map was generated using the reference map by KEGG. CO represents the entry number of chemicals. C00065: Serine, C00188: threonine, C00037: glycine, C00078: tryptophan. The matched chemicals showed different heat map colors based on their P values. 
detection through constructing standard curves, 14 out of 17 candidates were confirmed. They are alanine, glycine, valine, isoleucine, serine, threonine, proline, methionine, tyrosine, tryptophan, ethyl 2-methylacetoacetate, levulinic acid, benzlmalonic acid and p-cresol. The 14 variables are significantly increased in urine of gastric cancer patients and revealed diagnostic values with AUC from 0.669 to 0.823. The combined AUC of 14 variables reached to 0.893 . We further got the cutoff value for each metabolite, which are useful as candidate biomarkers in clinic. Importantly, the 14 candidate metabolites also increased significantly in early gastric cancer patients. Compared to classic blood biomarkers of CEA, AFP, CA72-4, CA199 and CA12-5, the urinary metabolites showed better diagnostic values. Particularly, three metabolites could predict the patient' prognosis. Higher levels of proline, p-cresol and 4-hydroxybenzoic acid were associated with poorer prognosis. Our new findings suggest urinary metabolites could be an alternative for gastric cancer screening.

By metabolic pathways analysis, the pathway of glycine, serine and threonine metabolism were excessively activated in gastric carcinogenesis. Jain and colleagues ever assayed metabolites profiles from media of NCI-60 cancer cell lines and found disorder of glycine consumption, which is correlated with proliferation rate of cancer cells [33]. Hirayama and coworkers [34] reported increased levels of amino acids in colon and gastric cancer tissues. Mayers and coworkers reported elevation of serum branched-chain amino acids in human pancreatic adenocarcinoma [35]. Chan and colleague [4] also found elevated alanine in the urine of gastric cancer patients. Obviously, disturbed amino acids levels in cancer tissue or body fluids are common in gastric cancer. Increased amino acids may due to robust metabolic adaptations for oxidative stress and cell growth.

However, whether the identified metabolites are gastric-cancer-specific or not remains unknown. Some reports disclosed an elevated urinary p-cresol in colorectal cancer [13], valine in bladder cancer [36], proline in ovarian cancer [37], glycine, threonine and tyrosine in liver cancer $[18,38]$. Obviously, one single metabolite may not be specific for gastric cancer, but the multiple metabolites combination may increase the specificity for gastric cancer. Therefore, it is necessary to collect various types of cancer for further comparison study.

\section{MATERIALS AND METHODS}

\section{Collection of urine samples}

A $5 \mathrm{ml}$ urine sample from 293 individuals was collected in the early morning before breakfast at Ruijin hospital, School of Medicine, Shanghai Jiao Tong
University. Of the 293 subjects, 159 were subjected to primary gastric cancer while the other 134 were controls who visited the hospital for physical examination with age and gender-paired to the patients. Patients with benign digestive diseases such as gastritis, gastric ulcer, or with metabolic diseases such as diabetes, hyperthyroidism were excluded. All of cases enrolled in the research showed normal liver and kidney function by blood biochemistry assay. The malignant diagnosis was confirmed by histopathology after biopsy or operation. The gastric cancer patients with metastases were diagnosed by image examination. Tumors were staged according to the UICC/ AJCC TNM Classification (Version 7). None of the patients was on any neoadjuvant chemotherapy before surgical treatment. All samples were obtained under the informed consent. The urine was centrifuged at $3000 \mathrm{rpm}$ for $10 \mathrm{~min}$ after obtaining. The supernatant and sediment were aliquoted separately into eppendorf tubes and then stored at $-80^{\circ} \mathrm{C}$ until use. The samples were divided into two sets. One was for training set with 47 urine samples from healthy controls and 47 urine samples from gastric cancer patients. Another was for validating set with 87 urine samples from healthy controls and 112 urine samples from gastric cancer patients. The age, gender and tumor stage for all patients are provided in Table 6. This study was approved by Hospital Institutional Review Boards for human subject research.

\section{Chemicals}

ECF, pyridine, anhydrous ethanol, sodium hydroxide, chloroform, and anhydrous sodium sulfate were analytical grade from China National Pharmaceutical Group Corporation (Shanghai, China). The standard reagents of alanine (Ala), glycine (Gly), isoleucine (Ile), valine (Val), praline (Pro), serine (Ser), threonine (Thr), methionine (Met), tyrosine (Tyr), tryptophan (Try), hippuric acid, ethyl 2-methylacetoacetate, levulinic acid, benzylmalonic acid,4-hydroxybenzoic acid, p-cresol and benzil were purchased (J\&K Scientific, USA). L-2chlorophenylalanine (Shanghai Hengbai Biotech. Co. Ltd., China) was used as an internal quality standard. Ethyl 2-methylacetoacetate, 4-hydroxybenzoic acid, benzil and hippuric acid were diluted in the anhydrous ethanol. Tyrosine was diluted in hydrochloricacid. Others were prepared in the ultra pure water from a Milli-Q system (Millipore, USA).

\section{Sample derivatization}

The process of sample derivatization was conducted as described before [29]. In brief, diluted urine sample (urine: water $=1: 1, \mathrm{v} / \mathrm{v}$ ) or each $600-\mu 1$ aliquot of standard mixture was added to glass tube. After adding $100 \mu \mathrm{l}$ of 1-2-chlorophenylalanine $(0.1 \mathrm{mg} / \mathrm{ml}), 400 \mu \mathrm{l}$ of anhydrous 
ethanol, and $100 \mu \mathrm{l}$ of pyridine to the urine sample, 50 $\mu 1$ of ECF was added for first derivatization at $20.0 \pm$ $0.1^{\circ} \mathrm{C}$. The mixtures were sonicated at $40 \mathrm{KHz} / \mathrm{s}$ for 60 s. Subsequently, exaction was performed using $300 \mu \mathrm{l}$ of chloroform. The derivatization procedure was repeated with the addition of $50-\mu 1$ ECF into the aforementioned products. After the two success derivatization, the overall mixtures were vortexed for $30 \mathrm{~s}$ and centrifuged for $3 \mathrm{~min}$ at $3000 \mathrm{rpm}$. The aqueous layer was aspirated off, while the remaining chloroform layer containing derivatives were isolated and dried with anhydrous sodium sulfate and subsequently subjected to GC-MS analysis.

\section{GC-MS analysis}

The extracts of derivatization were analyzed with a7890A gas chromatograph coupled with a5975C mass spectrometer (Agilent technologies Inc, USA). A 1- $\mu$ l extract aliquot of the extracts was injected into a DB-5MS capillary column coated with $5 \%$ diphenyl cross-linked $95 \%$ dimethylpolysiloxane $(30 \mathrm{~m} \times 250 \mu \mathrm{m}, 0.25-\mu \mathrm{m}$ film thickness; Agilent J\&W Scientific, Folsom, CA) in the split mode $(3: 1)$. Either the injection temperature or the interface temperature was set to $260^{\circ} \mathrm{C}$; and the ion source temperature was adjusted to $200{ }^{\circ} \mathrm{C}$. Initial GC oven temperature was $80{ }^{\circ} \mathrm{C}$. Two-min after injection, the GC oven temperature was raised to $140{ }^{\circ} \mathrm{C}$ with $10{ }^{\circ} \mathrm{C} / \mathrm{min}$, to $240{ }^{\circ} \mathrm{C}$ at rate of $4{ }^{\circ} \mathrm{C} / \mathrm{min}$, to $280{ }^{\circ} \mathrm{C}$ with $10{ }^{\circ} \mathrm{C} / \mathrm{min}$ again, and finally held at $280^{\circ} \mathrm{C}$ for $3 \mathrm{~min}$. Helium was the carrier gas with a flow rate set at $1 \mathrm{ml} / \mathrm{min}$. The detection was conducted with electron impact ionization $(70 \mathrm{eV})$ in both the full scan mode and selective ion monitoring (SIM) scan mode $(30-550 \mathrm{~m} / \mathrm{z})$. We set the ion as follows: l-alanine $44 \mathrm{~m} / \mathrm{z}, \quad$-glycine $102 \mathrm{~m} / \mathrm{z}$, l-valine $144 \mathrm{~m} / \mathrm{z}$, l-serine $132 \mathrm{~m} / \mathrm{z}$, l-isoleucine $158 \mathrm{~m} / \mathrm{z}$, l-threonine $101 \mathrm{~m} / \mathrm{z}$, 1-proline $142 \mathrm{~m} / \mathrm{z}$, 1-methionine $61 \mathrm{~m} / \mathrm{z}$, l-tyrosine $107 \mathrm{~m} / \mathrm{z}$, l-tryptophan $130 \mathrm{~m} / \mathrm{z}$, ethyl 2-methylacetoacetate $74 \mathrm{~m} / \mathrm{z}$, levulinic acid $99 \mathrm{~m} / \mathrm{z}$, cresols $107 \mathrm{~m} / \mathrm{z}$, benzylmalonic acid $131 \mathrm{~m} / \mathrm{z}$, 4-hydroxybenzoic acid $121 \mathrm{~m} / \mathrm{z}$, benzil $105 \mathrm{~m} / \mathrm{z}$, hippuric acid $134 \mathrm{~m} /$ zandl-2-chlorophenylalanine $102 \mathrm{~m} / \mathrm{z}$.

\section{Data analysis}

TICs and fragmentation patterns were acquired using GC/MS ChemStation Software (Agilent Technologies, Palo Alto, CA, USA). In training set, the compound identification was performed by comparing the mass spectrum with a standard mass spectrum in the national institute of standards and technology mass spectra library (NIST) with a similarity of more than $70 \%$ and verified by available standard compounds. The relative peak area of each compound would be calculated as the level of corresponding compound. For the holistic treatment of these data, multivariate analysis was used to identify the metabolomics differences between the groups. The OPLS-
DA was carried out with SIMCA-p software (v 12.0; Umetrics), while SAM analysis was performed with MEV software (http://statweb.stanford.edu/ tibs/SAM/index. html). Regarding to OPLS-DA, the VIP is considered to be differentiating variables. VIP scores indicate the relative importance of each metabolite in a given OPLS-DA model. Metabolites with a VIP $>1.0$ are more influential and thus contribute more to discriminating disease groups [39]. By SAM analysis, the false discovery rate (FDR) was set at 0.01 and the permutation was set at 1000, while the delta value was set at 1.5. By Mann-Whitney $\mathrm{U}$ test, the metabolites with $P<0.01$ were selected as candidate biomarkers. We integrated the outputs from the three numerations and chose 17 metabolites as candidate biomarkers for subsequent validation.

In validating set, the levels of 17 candidate compounds were calculated by external standard method through constructing standard curves. The sample information, peak intensities and peak retention time are applied for pattern recognition. The concentration of 17 candidate compounds was expressed by median, interquartile range and box chart. The Mann-Whitney $\mathrm{U}$ test was used for variables analysis between different groups. The classical tumor markers of CEA, CA199, CA72-4, CA12-5 and AFP were examined by chemiluminescence immunoassay (CLIA, Abbott ARCHITECT i2000) or electrochemiluminescence immunoassay (ECLIA, Roche Ecl 2010) during routine blood examination. The ROC curve, AUC, binary logistic regression and Kaplan-Meier analysis were performed with SPSS software (v20, IBM, USA). Metabolic pathway and function analysis was performed by MetPA's tool, which is a web-based metabolomics tool for pathway analysis (http://metpa.metabolomics.ca/MetPA/faces/ Home.jsp).

\section{ACKNOWLEDGMENTS}

This project was partially supported by the Chinese National High Tech Program (2012AA02A504, 2012AA02A203), the National Natural Science Foundation of China (81172329 and 81372644), International Cooperative Project from Shanghai Science and Technology Commission (12410706400), the Innovation Foundation of Translational Medicine of Shanghai Jiao Tong University School of Medicine (15ZH1002, 15ZH3001 and 15ZH4001), the CrossInstitutes Research Fund of Shanghai Jiao Tong University (YG2015MS62) and Project of Biobank of Gastrointestinal Carcinoma of Shanghai Jiao Tong University School of Medicine. Chinese patent application No. 201310556922.6; 201310556941.9.

\section{CONFLICTS OF INTERESTS}

The authors declare that they have no competing 
financial interests.

\section{Authors' Contributions}

YNC, JZ, LG, LL, LX finished the experiments. JRW, ZFL, XYZ and PN carried out data analysis and models building. MY, JL J, JJ, JNZ, WC collected samples and clinical information. HSZ and YW provided experimental equipment. ZGZ and YYY participated in the concept and design of the study. YNC and YYY wrote the manuscript. All authors read and approved the final version of the manuscript.

\section{REFERENCES}

1. Torre LA, Bray F, Siegel RL, Ferlay J, Lortet-Tieulent J and Jemal A. Global cancer statistics, 2012. CA. 2015; 65:87108.

2. Dunn WB, Broadhurst DI, Atherton HJ, Goodacre R and Griffin JL. Systems level studies of mammalian metabolomes: the roles of mass spectrometry and nuclear magnetic resonance spectroscopy. Chemical Society reviews. 2011; 40:387-426.

3. Smedsgaard J and Nielsen J. Metabolite profiling of fungi and yeast: from phenotype to metabolome by MS and informatics. Journal of experimental botany. 2005; 56:273286.

4. Chan AW, Mercier P, Schiller D, Bailey R, Robbins S, Eurich DT, Sawyer MB and Broadhurst D. (1)H-NMR urinary metabolomic profiling for diagnosis of gastric cancer. British journal of cancer. 2016; 114:59-62.

5. Yu L, Aa J, Xu J, Sun M, Qian S, Cheng L, Yang S and Shi R. Metabolomic phenotype of gastric cancer and precancerous stages based on gas chromatography time-offlight mass spectrometry. Journal of gastroenterology and hepatology. 2011; 26:1290-1297.

6. Jobard E, Pontoizeau C, Blaise BJ, Bachelot T, ElenaHerrmann B and Tredan O. A serum nuclear magnetic resonance-based metabolomic signature of advanced metastatic human breast cancer. Cancer letters. 2014; 343:33-41.

7. Kim Y, Koo I, Jung BH, Chung BC and Lee D. Multivariate classification of urine metabolome profiles for breast cancer diagnosis. BMC bioinformatics. 2010; 11 Suppl 2:S4.

8. Gunther UL. Metabolomics Biomarkers for Breast Cancer. Pathobiology. 2015; 82:153-165.

9. Fan Y, Zhou X, Xia TS, Chen Z, Li J, Liu Q, Alolga RN, Chen Y, Lai MD, Li P, Zhu W and Qi LW. Human plasma metabolomics for identifying differential metabolites and predicting molecular subtypes of breast cancer. Oncotarget. 2016; 7:9925-9938. doi: 10.18632/oncotarget.7155.

10. Kyriakides M, Rama N, Sidhu J, Gabra H, Keun HC and ElBahrawy M. Metabonomic analysis of ovarian tumour cyst fluid by proton nuclear magnetic resonance spectroscopy.
Oncotarget. 2016; 7:7216-7226. doi: 10.18632/ oncotarget.6891.

11. Di Gangi IM, Mazza T, Fontana A, Copetti M, Fusilli C, Ippolito A, Mattivi F, Latiano A, Andriulli A, Vrhovsek U and Pazienza V. Metabolomic profile in pancreatic cancer patients: a consensus-based approach to identify highly discriminating metabolites. Oncotarget. 2016; 7:5815-5829. doi: 10.18632/oncotarget.6808.

12. Qiu Y, Cai G, Zhou B, Li D, Zhao A, Xie G, Li H, Cai S, Xie D, Huang C, Ge W, Zhou Z, Xu LX, Jia W, Zheng $\mathrm{S}$ and Yen $\mathrm{Y}$. A distinct metabolic signature of human colorectal cancer with prognostic potential. Clinical cancer research 2014; 20:2136-2146.

13. Cheng Y, Xie G, Chen T, Qiu Y, Zou X, Zheng M, Tan B, Feng B, Dong T, He P, Zhao L, Zhao A, Xu LX, Zhang $\mathrm{Y}$ and Jia W. Distinct urinary metabolic profile of human colorectal cancer. Journal of proteome research. 2012; 11:1354-1363.

14. Wang H, Wang L, Zhang H, Deng P, Chen J, Zhou B, Hu J, Zou J, Lu W, Xiang P, Wu T, Shao X, Li Y, Zhou Z and Zhao YL. (1)H NMR-based metabolic profiling of human rectal cancer tissue. Molecular cancer. 2013; 12:121.

15. Lin Y, Ma C, Liu C, Wang Z, Yang J, Liu X, Shen Z and $\mathrm{Wu}$ R. NMR-based fecal metabolomics fingerprinting as predictors of earlier diagnosis in patients with colorectal cancer. Oncotarget. 2016; 7:29454-64. doi: 10.18632/ oncotarget.8762.

16. Struck-Lewicka W, Kordalewska M, Bujak R, Yumba Mpanga A, Markuszewski M, Jacyna J, Matuszewski M, Kaliszan R and Markuszewski MJ. Urine metabolic fingerprinting using LC-MS and GC-MS reveals metabolite changes in prostate cancer: A pilot study. Journal of pharmaceutical and biomedical analysis. 2015; 111:351361.

17. Lucarelli G, Rutigliano M, Galleggiante V, Giglio A, Palazzo S, Ferro M, Simone C, Bettocchi C, Battaglia M and Ditonno P. Metabolomic profiling for the identification of novel diagnostic markers in prostate cancer. Expert review of molecular diagnostics. 2015; 15:1211-1224.

18. Shariff MI, Gomaa AI, Cox IJ, Patel M, Williams HR, Crossey MM, Thillainayagam AV, Thomas HC, Waked I, Khan SA and Taylor-Robinson SD. Urinary metabolic biomarkers of hepatocellular carcinoma in an Egyptian population: a validation study. Journal of proteome research. 2011; 10:1828-1836.

19. Shao Y, Zhu B, Zheng R, Zhao X, Yin P, Lu X, Jiao B, $\mathrm{Xu} \mathrm{G}$ and Yao Z. Development of urinary pseudotargeted LC-MS-based metabolomics method and its application in hepatocellular carcinoma biomarker discovery. Journal of proteome research. 2015; 14:906-916.

20. Carrola J, Rocha CM, Barros AS, Gil AM, Goodfellow BJ, Carreira IM, Bernardo J, Gomes A, Sousa V, Carvalho L and Duarte IF. Metabolic signatures of lung cancer in biofluids: NMR-based metabonomics of urine. Journal of proteome research. 2011; 10:221-230. 
21. Chen Y, Ma Z, Li A, Li H, Wang B, Zhong J, Min L and Dai L. Metabolomic profiling of human serum in lung cancer patients using liquid chromatography/hybrid quadrupole time-of-flight mass spectrometry and gas chromatography/ mass spectrometry. Journal of cancer research and clinical oncology. 2015; 141:705-718.

22. An YJ, Cho HR, Kim TM, Keam B, Kim JW, Wen H, Park CK, Lee SH, Im SA, Kim JE, Choi SH and Park S. An NMR metabolomics approach for the diagnosis of leptomeningeal carcinomatosis in lung adenocarcinoma cancer patients. International journal of cancer. 2015; 136:162-171.

23. Mathe EA, Patterson AD, Haznadar M, Manna SK, Krausz KW, Bowman ED, Shields PG, Idle JR, Smith PB, Anami K, Kazandjian DG, Hatzakis E, Gonzalez FJ and Harris CC. Noninvasive urinary metabolomic profiling identifies diagnostic and prognostic markers in lung cancer. Cancer research. 2014; 74:3259-3270.

24. Jin X, Yun SJ, Jeong P, Kim IY, Kim WJ and Park S. Diagnosis of bladder cancer and prediction of survival by urinary metabolomics. Oncotarget. 2014; 5:1635-1645. doi:10.18632/oncotarget.1744.

25. Chan EC, Pasikanti KK, Hong Y, Ho PC, Mahendran R, Raman Nee Mani L, Chiong E and Esuvaranathan K. Metabonomic profiling of bladder cancer. Journal of proteome research. 2015; 14:587-602.

26. Chen JL, Fan J and Lu XJ. CE-MS based on moving reaction boundary method for urinary metabolomic analysis of gastric cancer patients. Electrophoresis. 2014; 35:10321039.

27. Liang Q, Wang C and Li B. Metabolomic Analysis Using Liquid Chromatography/Mass Spectrometry for Gastric Cancer. Applied biochemistry and biotechnology. 2015; 176:2170-2184.

28. Jung J, Jung Y, Bang EJ, Cho SI, Jang YJ, Kwak JM, Ryu do H, Park S and Hwang GS. Noninvasive diagnosis and evaluation of curative surgery for gastric cancer by using NMR-based metabolomic profiling. Annals of surgical oncology. 2014; 21 Suppl 4:S736-742.

29. Qiu Y, Su M, Liu Y, Chen M, Gu J, Zhang J and Jia W. Application of ethyl chloroformate derivatization for gas chromatography-mass spectrometry based metabonomic profiling. Analytica chimica acta. 2007; 583:277-283.

30. Chen Y, Zhu Z and Yu Y. Novel methodologies in analysis of small molecule biomarkers and living cells. Tumour biology. 2014; 35:9469-9477.
31. Youden WJ. Index for rating diagnostic tests. Cancer. 1950; 3:32-35.

32. Society AC. Survival rates for stomach cancer, by stage [Online]. American Cancer Society. 2015; Available at http:/www.cancer.org/cancer/stomachcancer/detailedguide/ stomach-cancer-survival-rates.

33. Jain M, Nilsson R, Sharma S, Madhusudhan N, Kitami T, Souza AL, Kafri R, Kirschner MW, Clish CB and Mootha VK. Metabolite profiling identifies a key role for glycine in rapid cancer cell proliferation. Science. 2012; 336:10401044.

34. Hirayama A, Kami K, Sugimoto M, Sugawara M, Toki N, Onozuka H, Kinoshita T, Saito N, Ochiai A, Tomita M, Esumi H and Soga T. Quantitative metabolome profiling of colon and stomach cancer microenvironment by capillary electrophoresis time-of-flight mass spectrometry. Cancer research. 2009; 69:4918-4925.

35. Mayers JR, Wu C, Clish CB, Kraft P, Torrence ME, Fiske BP, Yuan C, Bao Y, Townsend MK, Tworoger SS, Davidson SM, Papagiannakopoulos T, Yang A, Dayton TL, Ogino S, Stampfer MJ, et al. Elevation of circulating branched-chain amino acids is an early event in human pancreatic adenocarcinoma development. Nature medicine. 2014; 20:1193-1198.

36. Pasikanti KK, Esuvaranathan K, Ho PC, Mahendran R, Kamaraj R, Wu QH, Chiong E and Chan EC. Noninvasive urinary metabonomic diagnosis of human bladder cancer. Journal of proteome research. 2010; 9:2988-2995.

37. Jiang $\mathrm{T}$, Lin $\mathrm{Y}$, Yin $\mathrm{H}$, Wang S, Sun Q, Zhang P and Bi W. Correlation analysis of urine metabolites and clinical staging in patients with ovarian cancer. International journal of clinical and experimental medicine. 2015; 8:1816518171.

38. Wu H, Xue R, Dong L, Liu T, Deng C, Zeng H and Shen $\mathrm{X}$. Metabolomic profiling of human urine in hepatocellular carcinoma patients using gas chromatography/mass spectrometry. Analytica chimica acta. 2009; 648:98-104.

39. Eriksson L, Byrne T, Johansson E, Trygg J and Vikstrom C. (2013). Multi- and Megavariate Data Analysis, Basic Principles and Applications. (Malmo, Sweden: MKS Umetrics AB). 